\title{
S81. Proffered paper: A new PD1-CD28 chimeric receptor overcomes PD-1-mediated immunosuppression in adoptive T cell therapy
}

\author{
S Grassmann, P Peters, Y Zeng, J Schmollinger, S Endres, S Kobold ${ }^{*}$ \\ From 1st Immunotherapy of Cancer Conference (ITOC1) \\ Munich, Germany. 12-14 March 2014
}

\begin{abstract}
Background
Although tumour-specific cytotoxic $\mathrm{T}$ cells are capable of killing tumour cells both in vitro and in vivo, treatment with adoptive $\mathrm{T}$ cell transfer does not lead to sufficient tumour regression without adjuvant therapy. Tumourpromoted $\mathrm{T}$ cell exhaustion and anergy have been proposed to contribute to this lack of efficacy. We and others have previously shown that programmed death receptor-1 (PD-1) upregulation is a hallmark of tumour infiltrating, adoptively transferred T cells. PD-1 and its ligand (PD-L1) constitute a major immunosuppressive axis driven by tumour cells. Disruption of this axis may hit an Achilles heel of tumour immune escape.
\end{abstract}

\section{Material and methods}

A PD1-CD28 chimeric receptor was cloned into the retroviral vector pMP71 and expressed in primary murine $\mathrm{T}$ cells specific for the model antigen ovalbumin (OT-1 cells). Functionality was addressed in vitro using ELISA and flow cytometry. In vivo, ovalbumin and PD-L1 overexpressing Panc02 cells (syngeneic pancreatic cancer cell line) were inoculated subcutaneously in immunocompetent female $\mathrm{C} 57 \mathrm{Bl} / 6$ mice. Mice $(\mathrm{n}=6$ per group) were treated twice i.v. with PD1-CD28 chimeric receptortransduced $\mathrm{T}$ cells or control $\mathrm{T}$ cells.

\section{Results}

In vitro, $\mathrm{PD}-1-\mathrm{CD} 28$ chimeric receptor-transduced primary $\mathrm{T}$ cells released 130 fold more interleukin-2 (IL-2) and 300 fold more interferon- $\gamma$ than untransduced or control-transduced $\mathrm{T}$ cells when stimulated with CD3 and PD-L1, demonstrating the functionality of the chimeric receptor $(\mathrm{p}=0.0014)$. In co-culture experiments with the Panc02 tumour cells, effective co-stimulation through PD1-CD28 was only seen in the presence of the TCR-recognized antigen ovalbumine and PD-L1. Upon blockade of MHC or PD-1, co-stimulation through the receptor was abrogated. Culture of transduced $\mathrm{T}$ cells in the presence of CD3 and PD-L1 increased cell numbers 4 fold and significantly increased viability of cells compared to untransduced or control-transduced T cells $(\mathrm{p}<$ $0.0001)$. In vivo, treatment of mice with an established (OVA and PD-L1 expressing) Panc02 subcutaneous tumour (mean tumour size at treatment onset $26 \mathrm{~mm}^{2}$ ) with PD1-CD28-transduced OT-1 slowed tumour growth compared to treatment with control-transduced OT-1 cells $(\mathrm{p}<0.001)$. This demonstrates the functionality of the chimeric receptor in an immunocompetent organism.

\section{Conclusions}

Adoptive T cells therapy with PD-1-CD28 chimeric receptor-transduced $\mathrm{T}$ cells is a promising approach to overcome PD-1-PD-L1-mediated tumour-induced anergy and immunosuppression.

Published: 12 March 2014

doi:10.1186/2051-1426-2-S2-I19

Cite this article as: Grassmann et al:: S81. Proffered paper: A new

PD1-CD28 chimeric receptor overcomes PD-1-mediated

immunosuppression in adoptive T cell therapy. Journal for

ImmunoTherapy of Cancer 2014 2(Suppl 2):119.

Division of Clinical Pharmacology, Munich, Germany 